\title{
Ce que le numérique fait à l'archéologie et aux archéologues. Un retour d'expériences et un projet de recherche en cours.
}

\author{
What digital is doing to archaeology and archaeologists. A feedback
}

and a research project in progress.

\author{
Christophe Tuffery ${ }^{1}$ \\ ${ }^{1}$ Inrap, Institut National de Recherches Archéologiques Préventives 121 rue d'Alésia, CS 20007 - F-75685 Paris \\ Cedex 14, France, christophe.tuffery@inrap.fr
}

RÉSUMÉ. L'article présente le travail en cours d'une thèse d'histoire à CY Cergy Paris Université, en partenariat avec l'Institut National du Patrimoine. Le déploiement en cours et de plus en plus rapide de dispositifs numériques en archéologie demeure faiblement interrogé, en particulier pour les activités de recherche dans cette discipline. Si les nouveaux moyens et les nouvelles pratiques numériques tentent de se rendre accessibles au plus grand nombre, elles laissent aussi de côté une partie des archéologues et révèlent des risques de fracture des collectifs de travail. Depuis une participation observante, l'auteur propose un travail réflexif pour une recherche épistémologique, d'histoire des sciences et techniques et de sociologie des organisations professionnelles de l'archéologie. Les effets étudiés concernent l'archéologie comme discipline et les archéologues comme ensemble de compétences individuelles, de pratiques collectives et d'identités professionnelles. Les matériaux d'étude sont constitués par de nombreuses observations et retours d'expérience depuis plus de dix ans dans le domaine de l'acquisition des données archéologiques de terrain, au sein de l'Institut national de recherches archéologiques préventives (Inrap), et dans plusieurs projets collectifs de recherche pluridisciplinaires.

ABSTRACT. The paper presents the ongoing work of a PhD in history at CY Cergy Paris University, in partnership with the Institut National du Patrimoine. The current and increasingly rapid deployment of digital devices in archaeology remains poorly questioned, especially for research activities in this discipline. While the new digital tools and practices attempt to make themselves accessible to the greatest number of people, they also leave out some archaeologists and reveal risks of fracture in work collectives. From an observant participation, the author proposes a reflexive research work in epistemological, history of science and technology and sociology of professional archaeological organisations. The effects studied concern archaeology as a discipline, and archaeologists as a set of individual skills, collective practices and professional identities. The study material is made up of numerous observations and feedback from more than ten years of experience in the field of archaeological field data acquisition at the Institut national de recherches archéologiques préventives (Inrap) and in several collective multidisciplinary research projects.

MOTS-CLÉS. numérisation, recherche, archéologie, multidisciplinarité, dispositifs techniques, réflexivité, épistémologie, histoire des sciences et techniques, sociologie des organisations professionnelles, gouvernementalité.

KEYWORDS. digitization, research, archaeology, multidisciplinarity, technical devices, epistemology, reflexivity, history of science and technology, sociology of professional organizations, governmentality.

\section{Introduction : l’archéologie et le numérique : une histoire déjà ancienne}

L'archéologie est la science qui étudie les traces de l'activité des hommes, à travers leurs différentes archives matérielles (structures archéologiques, biens mobiliers et immobiliers, œuvres d'art, archives sonores et visuelles, etc.), replacées dans le contexte de leur découverte qui permet de leur donner sens.

Aujourd'hui, l'archéologie fait partie des sciences humaines et sociales qui sont progressivement concernées par le déploiement de dispositifs numériques et elle participe de cet ensemble en voie de structuration que constituent les « humanités numériques » [MOUNIER 12] [LE DEUFF 14]. 
Dans cet article, le terme de «dispositif numérique » désigne " tout ce qui a, d'une manière ou une autre, la capacité de capturer, d'orienter, de déterminer, d'intercepter, de modeler, de contrôler et d'assurer les gestes, les conduites, les opinions et les discours des êtres vivants » [AGAMBEN 14].

A la suite de nombreux auteurs, nous avons choisi de considérer les outils numériques non seulement du point de leur matérialité (ordinateurs, tablettes, smartphones, écrans, claviers, appareils d'acquisition de données nativement numériques, appareils d'impression sur supports papier ou impression 3D sur divers types de matériaux, casque de réalité immersive, etc.), mais comme des ensembles associant les matériels et les individus (chercheurs, ingénieurs, techniciens, personnels administratifs, médiateurs, publics divers, etc.) s'ancrant dans des pratiques professionnelles, culturelles, scientifiques, de médiation, elles-mêmes encadrées par des des normes formelles (codes, lois, règlements) ou plus discrètes (usages admis, reconnaissance par les pairs).

Si l'usage de l'informatique en archéologie a commencé dès les années 1950 avec les travaux de Jean-Claude Gardin [GARDIN 91], c'est surtout au cours des années 1990 que la production des informations de terrain a commencé à s'appuyer sur des dispositifs numériques. En France, on peut mentionner les systèmes pionniers Syslat [PY 91] et Arkeoplan [BUCHSENSCHUTZ 89] [GRUEL 90]. Depuis, de nombreuses autres applications ont été développées avec des solutions informatiques très variées : carnets électroniques de terrain des topographes, tableurs, bases de données, SIG, etc. [DESACHY 08] [DESACHY 16]. C'est le cas notamment au sein de l'Inrap, le plus grand organisme de recherches archéologiques préventives en France. Pour notre part, depuis 10 ans, nous avons contribué à l'inventaire et à l'étude de plusieurs dizaines des outils numériques utilisés à l'Inrap, en vue de leur harmonisation [KOEHLER 12]. Nous avons aussi développé plusieurs applications d'enregistrement de terrain, comme l'application EDArc pour la saisie des données d'opérations archéologiques de l'Inrap [TUFFERY 19a], et d'autres applications en situation de mobilité pour les besoins des Projets Collectifs de Recherche pourtant sur l'inventaire et l'étude des géoressources en silicites pour la préhistoire (PCR Réseau de lithothèques et GDR SILEX) [FERNANDES 13].

Nous proposons ici de mettre en perspective notre expérience depuis une position de participation observante qui va au-delà de l'observation participante [SOULE 07]. Si elle peut souffrir parfois de biais méthodologiques, la participation observante présente un intérêt majeur par rapport à l'observation participante, en ce qu'elle est une mise en perspective et une réflexivité d'expériences et d'observations de terrain faites par un acteur de l'organisation dans laquelle s'effectue l'observation. L'auteur de l'observation lui-même peut aussi être sujet de l'observation. Il a souvent un accès immédiat ou facilité aux aspects ou plus difficiles d'accès ou parfois invisibles pour l'observation participante.

En considérant les outils numériques utilisés en archéologie comme des dispositifs, nous avons souhaité nous interroger sur leurs usages, en particulier dans la recherche effectuée dans cette discipline. C'est l'objet de notre thèse d'histoire et d'épistémologie, engagée à l'automne 2019 à Cergy Paris Université, en partenariat avec l'Institut national de patrimoine.

\section{Un double point de vue archéologique pour interroger les dispositifs numériques en archéologie}

Pour interroger les effets du numérique en archéologie, nous proposons de développer un double point de vue « archéologique ».

D’une part, nous interrogeons le processus de « numérisation du monde » en nous inspirant de la méthode « archéologique » de Michel Foucault. Celle-ci consiste à révéler les soubassements largement impensés des sciences (plus particulièrement des sciences humaines) par l'étude de leur épistémès, c'est-à-dire de leurs conditions de possibilité (cf. infra). Nous proposons d'identifier en (c) 2021 ISTE OpenScience - Published by ISTE Ltd. London, UK - openscience.fr 
particulier comment ce processus de numérisation se traduit aujourd'hui pour l'archéologie, en inscrivant cette tentative dans une dimension historiographique de la discipline.

D'autre part, nous proposons à la communauté des archéologues, et à celle des informaticiens qui les accompagnent parfois, des pistes de réflexion sur les dispositifs numériques qu'ils conçoivent, développent et utilisent au quotidien, à des fins d'une mise en œuvre et d'usages raisonnés, rejoignant d'autres contributions et interrogations dans ce domaine [VERGNIEUX 18].

Sans en refaire ici toute l'histoire, que certains font remonter à la machine de Turing, voire à la Pascaline [DOUEIHI 13], nous proposons d'inscrire l'apparition et le déploiement des dispositifs numériques dans une histoire des sciences et des techniques. A ce titre, divers angles d'étude sont possibles.

Pour le philosophe Milad Doueihi, le numérique consiste en :

«une nouvelle manière de fabriquer de la mémoire et de l'interpréter (...) il nous oblige à repenser nos rapports avec ce qui est déjà mémorisé mais également à imaginer de nouvelles façons de préserver et exploiter nos productions purement numériques » [DOUEIHI 13].

Nous délaissons l'approche techniciste du numérique, traditionnellement présentée comme une innovation. Nous lui préférons une approche par les usages, centrée sur les objets et ce dont ils témoignent de la réelle adoption de dispositifs techniques par leurs utilisateurs, à la suite d'Edgerton [EDGERTON 13].

Pour l'archéologie, pas plus que pour les autres disciplines scientifiques, les dispositifs numériques ne doivent être déconnectés de leurs concepteurs, de leurs fabricants, de leurs utilisateurs, de leurs usages, des pratiques dans lesquelles ils sont mis en œuvre. Ces dispositifs sont conçus, fabriqués, mis en scène, utilisés, adaptés, remplacés, documentés et institutionnalisés par des organisations sociales, professionnelles ou autres. Ils sont le fruit de pratiques socialisées et participent de l'identité des métiers et des individus. Comme pour tout dispositif techniques, les utilisateurs se les approprient ou les rejettent, selon des itinéraires très variables des couples acteursobjets. Les usages effectifs témoignent de l'écart, parfois important, entre le projet initial du concepteur du dispositif et ce que son utilisateur en fait véritablement.

«il nous faut sans arrêt effectuer l'aller-retour entre le concepteur et l'utilisateur, entre l'utilisateur-projet du concepteur et l'utilisateur réel, entre le monde inscrit dans l'objet et le monde décrit par son déplacement » [AKRICH 10].

\section{La numérisation comme une condition de possibilité de la recherche archéologique}

En prenant l'archéologie comme champ disciplinaire d'étude, nous proposons d'interroger certaines des pratiques de la recherche dans ce domaine, en nous appuyant sur la méthode développée par le philosophe Michel Foucault dans la " période archéologique » de son œuvre au cours des années 1960. Foucault a proposé d'interroger les sciences humaines sur leurs épistémès, c'est-à-dire les conditions de possibilité des savoirs dans une culture et pour une époque donnée, qui se traduisent par des dispositifs (pratiques discursives et non-discursives) qui sont structurés comme des strates. L'analogie entre cette méthode et l'approche stratigraphique des archéologues apparait aussitôt.

Pour Foucault, l'épistémè consiste en :

"l'ensemble des relations pouvant unir, à une époque donnée, les pratiques discursives qui donnent lieu à des figures épistémologiques, à des sciences, éventuellement à des systèmes 
formalisés ; le mode selon lequel, dans chacune de ces formations discursives, se situent et s'opèrent les passages à l'épistémologisation, à la scientificité, à la formalisation » [FOUCAULT 69].

La méthode archéologique foucaldienne vise précisément à identifier les dispositifs en œuvre dans la construction des savoirs par l'analyse de « l'archive », et à en révéler les significations autres que celle que se donnent les savoirs, devenus invisibles en se masquant derrière l'évidence apparente de la raison. Foucault établit ainsi une analogie entre sa méthode et la méthode stratigraphique des archéologues qui consiste à mettre au jour les « archives du sol », à en dégager les relations et à en révéler la véritable signification qui serait masquée par l'évidence de la surface du sol.

Partant de la notion d'épistémè, l'usage du numérique dans la recherche archéologique peut être considéré comme l'une de ses conditions de possibilité.

Pour le chercheur en informatique Gérard Berry, à la suite d'autres auteurs, parfois promus par de grands acteurs économiques du secteur de l'informatique [HEY 09], le numérique constituerait un changement de paradigme car il s'agirait d' " une façon de penser et d'agir radicalement différente $\gg$ [BERRY 19].

Avant de pouvoir affirmer que l'usage du numérique constitue un réel changement de paradigme, il conviendrait d'en apporter des preuves multiples et convergentes, en démontrant que le numérique relève bien d'une « révolution scientifique » au sens donnée à cette notion par Thomas Kuhn [KUHN 08], à la suite d'Alexandre Koyré. Or, toute " révolution scientifique » intervient parce qu'il y a d'abord une crise dans ce que Kuhn appelle la « science normale », c'est-à-dire les représentations des savants à une époque donnée.

Pour pouvoir démontrer que le numérique constitue une révolution, il manque encore d'une part d'un certain recul qu'impose l'analyse historique qui a besoin d'une certain recul, et d'autre part de cas pratiques analysés, d'expériences étudiées selon des protocoles éprouvés. Sans cela, la « révolution numérique » relève encore d'une sorte de pensée magique, voire d'une idéologie qui ne favorise pas l'esprit critique [DACHEUX 18]. En cela, il est un sujet de prédilection pour les discours mythologiques, parfois alimentés par la science-fiction depuis près d'un siècle [BOULLIER 19]. A notre sens, la prétendue " révolution numérique » relève d'une série de ce que Gilbert Simondon appelle des « perfectionnements ». Il reste à identifier ce que sont, dans l'histoire du numérique, les «perfectionnements majeurs » et les «perfectionnements mineurs ». Les premiers ressortissent à une véritable rupture dans une lignée technique, par une ou plusieurs mutations orientées par des intentions. Les seconds n'ont que l'apparence des changements et ne présentent aucun bond technique, «aucune frontière tranchée par rapport à ce faux renouvellement que le commerce exige pour pouvoir présenter un objet récent comme supérieure aux plus anciens » [SIMONDON 89].

En prenant le domaine de l'archéologie comme objet d'observation, nous pensons utile d'interroger les usages des dispositifs numériques plutôt que de ne présenter que les outils (et de risquer de les survaloriser) numériques (matériels, logiciels, données, etc.), rejoignant en cela les réflexions proposées par l'archéologue britannique Jeremy Huggett depuis une vingtaine d'années [HuGGETT 00].

Comme l'évoque Anne Lehoërff, Vice-Présidente du Conseil National de la Recherche Archéologique (CNRA), le numérique concerne plusieurs des méthodes de l'archéologie depuis le terrain jusqu'aux différentes formes de représentation et de publication des résultats de la recherche archéologique [LERHOËFF 19].

Prenons comme premier exemple celui du remplacement progressif des carnets de terrain de l'archéologue par des applications numériques. Traditionnellement, les archéologues utilisent des 
carnets au format livre de poche avec ou sans élastique, couverture plastifiée ou pas, avec ou sans carreaux, parfois des fiches types pré-imprimées, rangées dans des classeurs d'enregistrement. Dans l'extrême majorité des cas, les carnets de terrain sont propres à l'archéologue. Ils lui appartiennent et lui seul les renseigne. Ses notes, ses commentaires, ses ajouts, ses modifications sur ses notes, ses schémas, ses observations et ses premières interprétations de terrain y sont consignées d'une façon qui lui est particulière et que, parfois, lui seul peut relire et comprendre. L'importance des carnets de terrain pour l'archéologie a été soulignée par Françoise Waquet [WAQUET 15] [WAQUET 19]. Ces objets matériels sont indétachables de la construction des savoirs. Longtemps, les émotions des chercheurs ne devaient pas être consignées, présentes dans les carnets de terrain, devaient disparaître des publications scientifiques, comme si les chercheurs se devaient de rester totalement extérieurs, neutres, insensibles aux objets de recherche. La dimension sensible et subjective devait être censurée, et n'avait pas le droit d'être assumée ni revendiquée par les chercheurs. Or les carnets de terrain témoignent d'une relation privilégiée entretenue par leurs auteurs avec leurs objets de recherche tout au long du processus d'observation sur le terrain. Ils disent les émotions et la subjectivité des chercheurs.

Ces constats ont pu être faits aussi bien pour l'archéologie que pour l'anthropologie, l'ethnologie, l'écologie, la géologie, la géographie et dans toutes les disciplines ou l'observation de terrain est centrale dans la construction des savoirs [BLANCKAERT 96]. Ce n'est que vers la fin du XXème siècle que la dimension émotionnelle des savoirs a pu commencer à s'exprimer dans les publications scientifiques. Mais la prétention à la neutralité et la mise à distance des émotions par les chercheurs demeure encore largement la norme des publications scientifiques.

Lorsque nous avons interrogé plusieurs dizaines d'archéologues, de l'Inrap ou d'autres institutions, beaucoup d'entre eux ont souligné que leurs carnets n'avaient pas vocation à être publiés, ni même versés au titre de la documentation de fouille. Ils les considèrent souvent comme des brouillons, des documents sans intérêt, qu'eux seuls peuvent relire, comprendre et exploiter. Cette auto-limitation de la portée de ces documents témoigne du désir de leurs auteurs de considérer ces carnets comme des documents très personnels, un peu à l'image du journal intime, que seul son propriétaire peut ouvrir, remplir, relire. Il est le seul qui peut décider un jour peut-être de lever l'interdiction à être diffusé voire publié, qui prévaut par défaut sur ces documents.

Or, en France, les carnets de terrain relèvent de la documentation de fouille. A ce titre doivent être versés au même titre que toute la documentation produite lors d'une opération de fouille réalisée dans le cadre d'une mission de service publique. Si les pratiques tendent à évoluer dans ce cadre réglementaire, il n'en reste pas moins que dans les pratiques, des archéologues continuent à tenir leur carnet de terrain sans les verser à l'Etat à l'issue de leurs travaux.

Avec l'émergence des carnets de terrain sous forme numérique, se pose la question des informations que les archéologues acceptent d'y inscrire, en sachant que les fichiers résultant de l'usage de ces dispositifs ont vocation à être lus par d'autres que leurs auteurs.

Les applications d'enregistrement de terrain sur lesquelles nous avons eu l'occasion de travailler, ont notamment comme principe commun de pouvoir être utilisées par tous les intervenants sur une opération archéologique ou travaillant sur une même thématique de recherche et s'affranchir, en apparence du moins, de toute forme de subjectivité des chercheurs. Avec ces dispositifs, l'archéologue ne peut plus s'exprimer de la même façon ni enregistrer exactement les mêmes types d'informations qu'avec les carnets de terrain traditionnels. Les notes, les commentaires, ne peuvent plus y être écrits ni les schémas y être dessinés de la même façon. Pour autant, l'usage de dispositifs numériques pour l'enregistrement des données d'observation et d'interprétation des archéologues, reste marginal par rapport au volume total des opérations archéologiques réalisée en France chaque année. 
En 2019-2020, nous avons pu recueillir et étudier plusieurs carnets de terrain et journaux de fouille, certains datant d'une cinquantaine d'années, d'autres plus récents, d'autres actuels. L'étude de ces documents a fait l'objet d'un mémoire de master 2 à l'Ecole des Hautes Etudes en Sciences Sociales $^{1}$ [TUFFERY 20].

Dans ce travail, il a été possible d'identifier les étapes de la construction des savoirs archéologiques sur le terrain et de révéler que les archéologues entretiennent entre eux et avec leurs objets de recherche des relations multiples, complexes où la sensibilité et l'expression des émotions ont leur place.

Si, par principe, l'enregistrement numérique de terrain permet de produire des archives nativement numériques dès le terrain, il implique aussi une reconfiguration dans la nature et dans la forme des archives de fouilles. L'expression de la subjectivité des archéologues semble être moindre et celle des émotions ne plus pouvoir y trouver sa place. Avec l'essor des dispositifs numériques, il semble qu'une certaine nouvelle conception de l'archéologie se déploie.

La simulation, en plan ou en vue perspective, peut être prise comme un second exemple des domaines de la recherche en archéologie concernés par l'usage de dispositifs numériques, en particulier pour la restitution en 3D de vestiges archéologiques (biens mobiliers), parfois en élévation (biens immobiliers). Dans ce domaine, le numérique s'est imposé de façon massive depuis une dizaine d'années, soit pour produire des figures statiques, soit pour créer des environnements virtuels interactifs, comme par exemple ceux que proposent les centres d'interprétation des grottes de Lascaux et de Chauvet .

Mais, ainsi que l'affirme la chercheuse Sylvie Eusèbe, spécialiste de ce sujet à l'Inrap :

« il ne faudrait pas céder à la facilité en s'en remettant à la représentation automatique proposée par les machines, se laisser ainsi «déresponsabiliser », et renoncer à l'interprétation de ces images sous prétexte que leur aspect photo-réaliste les rend intelligibles par tous. "La donnée n'est pas la pensée » comme titrait récemment une rencontre interdisciplinaire ; la donnée n'est pas le savoir» [EUSEBE 19].

Ces divers dispositifs numériques utilisés en archéologie marquent une évolution certaine des outils mais pas des processus intellectuels ni des représentations des archéologues à propos de leur objet d'étude. Pour Huggett, s'il y a un changement de paradigme lié au numérique en archéologie, celui-ci serait à rechercher davantage dans les effets de la production massive de données numériques (Big Data) sur les modalités de production et de traitement des savoirs archéologiques, plutôt que dans la définition et l'usage des catégories traditionnelles de ces savoirs [HUGGETT 20].

En nous démarquant d'une opposition dualiste et trop simpliste entre les dispositifs traditionnels et ceux qui relèvent des techniques numériques, nous pensons plus utile de constater que la discipline archéologique conserve une certaine continuité dans ses objectifs et ses grands principes méthodologiques face aux usages grandissants de dispositifs numériques. Ainsi, l'archéologie n'exclut pas aujourd'hui une coexistence entre les modes traditionnels de production et diffusion de la documentation archéologique (carnets de terrain, rapport de fouilles, monographies de sites, synthèses régionales, thématiques, chronologiques, etc.) et leurs équivalents numériques (carnets électroniques de terrain, bases de données ciblées ou généralistes, rapports de fouille en ligne, sites cartographiques, etc.).

Au-delà des outils numériques eux-mêmes, il nous semble utile d'interroger les effets des dispositifs numériques sur l'archéologie comme discipline et les archéologues dans leurs pratiques

${ }^{1}$ http://www.ehess.fr/fr/parcours-histoire-sciences-techniques-et-savoirs 
de quotidiennes et dans le fonctionnement de leurs collectifs professionnels de travail. En quoi l'usage de ces dispositifs ne concerne pas seulement les habitudes de travail mais aboutit à une refonte des principes mêmes de la discipline ? En quoi l'évolution de ces dispositifs modifie-t-il le comportement, les pratiques, les discours et jusqu'aux institutions mêmes de l'archéologie ? La réponse à ces questions est peut-être, là aussi, à rechercher du côté de l'œuvre de Michel Foucault.

\section{Le numérique comme entreprise de gouvernementalité technique}

Comme tout dispositif technique, ceux du domaine du numérique relèvent d'une entreprise de gouvernementalité, dans le sens que Foucault donna à cette notion qui désigne d'une part "les institutions, les procédures, analyses et réflexions, les calculs et les tactiques qui permettent d'exercer cette forme bien spécifique, quoique très complexe de pouvoir qui a pour cible principale la population, pour forme majeure de savoir l'économie politique, pour instrument essentiel les dispositifs de sécurité » et d'autre part " la tendance, la ligne de force qui, dans tout l'Occident, n'a pas cessé de conduire, et depuis fort longtemps, vers la prééminence de ce type de "gouvernement" sur tous les autres: souveraineté, discipline, et qui a amené, d'une part, le développement de toute une série d'appareils spécifiques de gouvernement, et, d'autre part, le développement de toute une série de savoirs » [FOUCAULT 04].

Dans L'art de ne pas être trop gouverné, le philosophe Jean-Claude Monod évoque ce qu'il appelle le «panoptique numérique » en référence au panoptique de Michel Foucault, un dispositif de surveillance permanente des individus qui eux, ne peuvent savoir quand ni par qui ils sont surveillés [MONOD 19].

De leur côté, Antoinette Rouvroy et Thomas Berns proposent une extension de la notion de gouvernementalité, telle que définie par Michel Foucault, avec celle de "gouvernementalité algorithmique » [ROUVROY 09]. Parmi les formes que prend cette dernière, les auteurs soulignent l'usage des normes.

En archéologie, les pratiques des chercheurs se voient imposer de plus en plus l'usage de normes diverses, certaines formelles, d'autres plus informelles :

- normes des formats numériques à utiliser et qui doivent assurer l'interopérabilité technique et sémantique des données, par l'usage de formats ouverts,

- normes de « bonnes pratiques » qui visent à distinguer celles définies comme «bonnes » et donc «normales » par opposition à toutes les autres qui se retrouvent de fait qualifiées de pratiques « anormales »,

- normes du développement d'applications et de programmes informatiques qui proviennent non pas du domaine de l'archéologie mais de celui de l'informatique et des sciences de l'information (modélisation conceptuelle, génie logiciel, documentation du code, documentation développeur, documentation utilisateur, remontées et corrections de bugs, versionnement des applications, etc.).

Parmi les nouvelles normes qui vont progressivement s'imposer à la recherche, en archéologie comme dans tout autre discipline, se trouvent les plans de gestion des données (PGD). Ces plans imposent que les données de recherche produites sur financements publics, du moins certains d'entre eux, s'inscrivent dans un cadre descriptif prédéfini, permettant la réutilisation de ces données selon les principes FAIR $^{2}$. Sans vouloir contester fondamentalement leur intérêt pour les divers acteurs concernés de la recherche, de telles pratiques normalisées peuvent être considérées

\footnotetext{
${ }^{2}$ Findable, Accessible, Retrievabe, Interoperable
} 
comme les moyens d'une gouvernementalité technique et pas seulement comme ceux d'une exigence accrue de scientificité.

Toutes ces normes, très codifiées, « disciplinarisent » les pratiques de recherche auxquelles elles s'imposent. Elles le font notamment par leurs effets sur les environnements dans lesquels les chercheurs exercent leur activité. Ainsi, ces normes imposent aux pratiques de recherche et aux chercheurs, de nouvelles conditions de possibilité et de nouveaux horizons affirmés comme incontournables.

Cette généralisation de la normalisation, toujours présentée au service du déploiement de la modernité et du mythe du progrès, a été dénoncée par sociologue français Jacques Ellul dans l'un de ses ouvrages majeurs :

«Il faut créer pour tout des normes, car la normalisation des données constitutives de la société, de l'être humain, permet seule l'application intégrale des techniques et en même temps permet seule l'universalisation » [ELLUL 12].

Pour Claude Dubar, les changements liés aux normes introduisent des perturbations dans les identités sociales et professionnelles :

«Le changement de normes, de modèles, de terminologie provoque une déstabilisation des repères, des appellations, des systèmes symboliques antérieurs. Cette dimension, même si elle est complexe et cachée, touche une question cruciale : celle de subjectivité, du fonctionnement psychique et des formes d'individualité ainsi mises en question » [DUBAR 00].

Parmi les autres enjeux du numérique, se trouve l'ambition d'une accélération de la temporalité dans laquelle s'exerce le travail des chercheurs. Le scénario idéal veut que les chercheurs profitent des outils numériques pour travailler plus vite, pour dégager du temps pour multiplier leurs activités, leur « productivité », devenue une notions envahissant le quotidien des chercheurs et l'aune de laquelle ils sont «évalués » [DEJOURS 03]. Cette accélération des pratiques de recherche s'appuie notamment sur l'accroissement des capacités de calcul et de fréquences des processeurs, mais aussi sur l'interopérabilité entre applications numériques ou encore entre outils ou objets dits « connectés ». Les communications entre individus sont plus rapides, les fichiers sont échangés plus vite, les données sont relues directement, etc. bref, tous ces principes sont au service du processus d'accélération dont le philosophe et sociologue allemand Hartmut Rosa a démontré les effets délétères :

«Les forces d'accélération de la société contemporaine, dans le passage du XXe au XXIe siècle, engendrent une redéfinition du rapport à soi-même, sur le plan individuel et collectif, c'est-à-dire des formes dominantes d'identité, de même que des formes de l'activité ou de l'organisation politique » [ROSA 10].

Harmut Rosa a récemment proposé la thèse que la numérisation participe du projet de « rendre le monde disponible » à tous, humains et machines, en tout lieu et à tout moment. Or, pour cet auteur, il y a urgence à ne pas prolonger cette entreprise mais à l'inverser pour permettre à l'humanité de trouver une autre relation avec la technique et pouvoir de nouveau entrer en résonnance avec elle [ROSA 20].

Tenter de révéler ainsi ce que cache le projet de numérisation de la recherche archéologique, c'est emprunter à la méthode archéologique foucaldienne son principal objectif qui est de faire ressortir l'épistémè des savoirs archéologiques pour l'époque actuelle. Cette épistémè est soutendue par une nouvelle conception du monde qui s'appuie sur des critères renouvelés de véridiction des pratiques actuelles de la recherche, qui témoignent de l'établissement du régime de vérité de ces pratiques. Le processus de numérisation du monde et de celui de la recherche en particulier, entraîne une 
reconfiguration des métiers, des pratiques et des identités professionnelles des chercheurs. Or, si comme l'affirme le PDG du CNRS, le processus en cours de numérisation de la recherche a l'ambition d'être au bénéfice du plus grand nombre, cela signifie, en creux, qu'il ne cherche pas à l'être au bénéfice de tous. Pourquoi cette ambition limitée ? Cette entreprise considère-t-elle possible de laisser certains chercheurs de côté ?

\section{Le numérique au bénéfice du plus grand nombre mais pas de tous}

Certes, pour une partie des archéologues, comme pour de nombreux autres chercheurs, l'usage de dispositifs numériques ouvre à des possibilités nouvelles pour faire évoluer leurs pratiques de recherche. Mais pour d'autres, la mise en œuvre de ces dispositifs les conduit à des évolutions contraintes et à dans certains cas, à des adaptations forcées à ces dispositifs [TUFFERY 19b] .

Ces changements de pratiques imposent aux archéologues de se former à divers dispositifs numériques (tablettes, appareils de topographie et de photographie numérique, outils d'étude non invasive des sols et des matériaux, etc.), mais aussi parfois à des langages et des méthodes propres au domaine de l'informatique et, prochainement, à de nouveaux modes de production et de diffusion de leurs connaissances comme les ontologies et leurs langages de description (RDF, XML, SKOS, etc.).

Plus encore, ce sont les pratiques des archéologues en matière de de stockage, d'archivage et de conservation des données archéologiques qui sont affectées par le déploiement d'outils numériques. Dans ces domaines, les archéologues sont invités à faire évoluer leurs pratiques et à faire preuve d'une vigilance accrue par rapport aux données qu'ils produisent, à multiplier les procédures de sauvegarde et donc les copies de leurs données, quitte à utiliser parfois des moyens personnels (stockage sur des disques durs externes, des clés USB, dans le «nuage » sur leur espace personnel sur des plateformes commerciales, etc.). En poussant à ce type de pratiques, l'usage des dispositifs numériques se traduit par des copies multiples des données sur des supports variés, personnels et professionnels, mais sans le souci d'en garantir pour autant l'intégrité, l'accessibilité, la sécurité ni la réutilisabilité.

Le sociologue Antonio Casilli [CASILLI 10] a montré que l'ordinateur est l'un des rares dispositifs techniques à modifier les trois espaces de la sphère domestique à la fois : espace physique (répartition, aménagement et usages des pièces, agencement des objets), espace technologique (outils, instruments utilisés pour répondre à des besoins), espace social (liens de sociabilité entre les individus par lesquels ils se construisent, s'individualisent, s'identifient). Il en est de même pour les dispositifs numériques qui modifient les trois espaces de la sphère professionnelle : l'espace physique (bureaux, laboratoire, terrain, lieux de rencontre entre individus, etc.), l'espace technologique (ordinateurs, tablettes, smartphones, serveurs, réseaux, etc.), espace social (liens de sociabilité par lesquels les individus s'affirment et construisent leur identité professionnelle).

Les moyens numériques s'insinuent partout dans les lieux de la recherche, ils créent une nouvelle territorialisation, ils imposent de nouveaux lieux, de nouvelles locaux (ex. des salles dédiée exclusivement aux serveurs informatiques), ils impliquent de mettre en place de nouvelles infrastructures (ex. réseaux informatiques spécifiques à la recherche comme le réseau RENATER), d'installer de nouveaux équipements (matériels informatiques en tous genres, armoires de brassage des réseaux, etc.). Cette reterritorialisation de la recherche participe d'une nouvelle configuration des « lieux de savoirs » pour reprendre le titre de l'ouvrage dirigé par Christian Jacob [JACOB 07] [ЈАСОВ 11]. En même temps qu'elle se déploie, la numérisation bénéficie de la miniaturisation des moyens techniques sur lesquels elle s'appuie : miniaturisation des circuit, remplacement des ordinateurs par des tablettes et des smartphones plus petits et plus légers. De façon paradoxale, la diminution de l'encombrement des moyens techniques de la numérisation pour un même niveau de 
capacité de calcul et de stockage, profite non pas à une diminution de l'occupation des lieux des dispositifs numériques mais au contraire à son entreprise d'extension dans l'espace. Cette conquête des espaces de travail des chercheurs par le numérique montre bien que la « dématérialisation » des savoirs modernes passe par une extension des espaces occupés par les moyens matériels nécessaires à cette dématérialisation.

Le numérique se traduit par des changements multiples et plus ou moins profonds sur ce que le sociologue Claude Dubar appelle les identités professionnelles, qui relèvent de deux catégories d'identification, « externes » (pour autrui) et « internes » (pour soi) [DUBAR 11]. Ces modifications concernent aussi bien les façons d'apprendre, que les compétences déjà acquises et d'autres à acquérir, ou encore les savoir-faire, les manières de faire, qui dépassent les simples compétences. Les savoirs et les savoir-faire voient leur valeur évoluer dans les dimensions scientifique, économique, symbolique, etc.

Le numérique intervient dans la «mise en scène » de la vie quotidienne pour reprendre la notion du sociologue Erving Goffman [GofFMAn 73]. Pour Goffman, les individus masquent leurs comportements par des éléments scéniques et matériels de leur environnement de travail qui constituent le « décor» (mobilier, objets, accessoires, etc.) dont le sociologue français Pierre Bourdieu a montré la fonction sociale de représentation et de distinction [BOURDIEU 79]. Les éléments matériels constituent la perspective «technique » de la mise en scène quotidienne de soi au travail. A côté de cette dimension matérielle, il en existe une autre, que Goffman appelle la « façade personnelle » et qui regroupe les attributs confondus avec la personne (ses façons de faire, de se mouvoir, de se saisir d'un dispositif technique, d'incorporer celui-ci dans ses gestes et ses postures qui sont liées à ses compétences, son sexe, sa physiologie, etc.) [MARCELLINI 99].

Les dispositifs numériques imposent aussi de nouvelles techniques du corps, pour reprendre l'expression de Marcel Mauss, de nouveaux gestes et de nouvelles postures.

«Ces «habitudes» varient non pas simplement avec les individus et leurs imitations, elles varient surtout avec les sociétés. Il faut y voir les techniques et l'ouvrage de la raison pratique collective et individuelle, là où on ne voit d'ordinaire que l'âme et ses facultés de répétition » [MAUSS 13].

Les corps des chercheurs doivent s'adapter à une numérisation croissante de leurs pratiques, ils doivent développer une capacité croissante à interagir avec des dispositifs numériques qui ne cessent de se multiplier. La dextérité des mains et des doigts est de plus en plus sollicitée pour naviguer, à travers des écrans tactiles, dans les menus et entre les fonctionnalités d'applications qui permettent d'enregistrer des informations ou d'interagir avec d'autres équipements. Les moyens numériques (ordinateurs, tablettes, smartphones, etc.) imposent donc aux individus des façons de s'en servir, de se mouvoir, ou de rester assis devant un ordinateur, un écran, un clavier, de prendre en main une souris, d'interagir avec un écran par une interface dite « homme-machine », de placer des objets à numériser dans des machines dédiées pour en faire la numérisation en 3D, d'installer des cibles sur les sols de sites archéologiques ou des sphères sur les parois d'art rupestre pour en faire un relevé en photogrammétrie, etc. Ces relations étroites entre les corps des chercheurs, leurs dispositifs matériels et leurs usages a été largement décrite par F. Waquet dans ce qu'elle appelle l'ordre matériel des savoirs qui n'est jamais uniquement matériel [WAQUET 15].

\section{Pour une mise en perspective}

Notre projet de recherche consiste à tenter de décoder ce que les appareils, les codes informatiques et les algorithmes font à une expérience de recherche dans une science humaine comme l'archéologie. Entre questionnements épistémologiques, sociologiques, historiographiques et anthropologiques, nous avons tenté de décrire et d'interroger les effets de la "culture numérique » (C) 2021 ISTE OpenScience - Published by ISTE Ltd. London, UK - openscience.fr 
[CARDON 19] sur des pratiques de travail de recherche et sur les identités professionnelles des chercheurs.

En aucune manière, le numérique ne peut se porter candidat au « solutionnisme technologique » qu'Evgueny Morozov, chercheur, journaliste et essayiste, spécialiste des implications politiques et sociales de la technologie, a déjà largement critiqué (Morozov, 2014), tout comme l'ont fait de nombreux auteurs en France [SADIN 16] [BIAGINI 12] [CALAN 19] [FILIPPOVA 19].

Certes, l'usage d'outils informatiques au service des pratiques de la recherche en archéologie fait quotidiennement la démonstration de ses bénéfices. Mais, comme tous les dispositifs techniques, ceux utilisés dans ce domaine ne sont pas neutres. Ils portent en eux leur généalogie, leur origine, etc. Ils ne s'arrogent pas par eux-mêmes leurs propres conditions de possibilité, qui sont fondamentalement liées au contexte socio-techno-culturel des organisations humaines dans lesquelles s’inscrivent ces dispositifs numériques et leurs utilisateurs.

A l'occasion de l'établissement du plan stratégique de l'Inria 2013-2017, le philosophe et historien des sciences Michel Serres, a suggéré que les acteurs de l'informatique soient sensibilisés aux sciences sociales.

«Autrement dit, l'informatique produit des réseaux de relations inédites et des institutions à l'état naissant, des individus originaux et des collectifs insolites. [...] Non seulement pour l'avenir des recherches propres à Inria, mais aussi pour le futur de nos sociétés, peut-être vaudrait-il mieux que les artisans de l'informatique forment leurs propres chercheurs aux sciences sociales et aux questions éthiques, quitte à les remodeler, plutôt que d'aller chercher dans ces disciplines telles qu'elles existent aujourd'hui, des chercheurs autrement formatés » [SERRES 13].

Cette suggestion du philosophe pourrait conduire à une prise en compte par des acteurs de l'informatique aux différentes dimensions que posent le processus de numérisation du monde qui ne relève pas uniquement d'un projet scientifique ou technologique mais bien anthropologique puisqu'il concerne une nouvelle représentation du monde que ce processus implique.

Avec l'historien et anthropologue François-Xavier Petit, nous pensons que :

«Il est temps que la technologie numérique prenne conscience de son pouvoir social (...). Le numérique est matériel. Il a des frontières, une sociologie. Il est temps de le reconnecter avec le sol social » [PETIT 19].

Le risque n'est pas nul, en effet, de voire certaines communautés de chercheurs, notamment en archéologie, faire une confiance aveugle aux machines et à leurs algorithmes. Ceux-ci n'ont de cesse de vouloir rendre plus rapides, plus faciles, plus fluides, plus liquides, les tâches de travail, au nom d'une rationalisation sans fin. Mais quel sens peut encore prendre le travail si celui-ci ne connaît plus aucune limite dans l'accélération du temps dans lequel il prend place ? Comment l'expérience humaine peut-elle se construire de façon supportable si le sens donné au travail se dissout dans des pratiques où les liens de socialisation sont fragilisés ? Jusqu'à quel point une pratique de recherche en science humaine peut-elle s'adosser à des dispositifs techniques, dans une sorte de croyance sans limite dans le pouvoir des techniques numériques [GOLLAC 00], qui confine parfois à un « fétichisme technologique » [HUGGETT 04] ?

En reprenant le titre de l'ouvrage de J-C. Monod [MONOD 19], gageons que pour l'archéologie, comme pour les autres domaines de la recherche française, l'art de ne pas se faire trop gouverner pourrait passer par celui de ne pas se faire trop numériser... ni trop programmer. 


\section{Bibliographie}

[AKRICH 10] AKRICH M., «Comment décrire les objets techniques ? », Techniques \& Culture, $\mathrm{n}^{\circ}$ 54-55, 2010, DOI : https://doi.org/10.4000/tc.4999

[Agamben 14] Agamben G., Qu'est-ce qu'un dispositif ? Rivages poches, Paris, 2014

[BIAGINI 12] Biagini E., L'emprise numérique. Comment Internet et es nouvelles technologies ont colonisé nos vies. Ed. L’Echappée, Paris, 2012.

[BLANCKAERT 96] Blanckaert C., Le terrain des sciences humaines. Instructions et enquêtes (XVIIIè-XXè siècle). L'Harmattan, Paris, 1996.

[Boullier 19] Boullier D., Sociologie du numérique. Armand Colin, Paris, 2019.

[Bourdieu 79] Bourdieu P., La distinction. Critique sociale du jugement. Les éditions de minuit, Paris, 1979.

[BUCHSENSCHUTZ 89] BUCHSENSCHUTZ O. Expérimentations sur le site du Mont-Beuvray. Le courrier du CNRS, 73, p.30, Paris, 1989.

[CAlan J. de 19] Calan J. De et Cauchard J., Remède contre l'hystérie numérique. Pourquoi la " révolution digitale » n'est pas une révolution. Robert Laffont, Paris, 2019.

[CARDOn 19] CARDON D., Culture numérique. Presses de la Fondation Nationale des Sciences Politiques, Paris, 2019.

[CASILli 10] CASILli A., Les Liaisons numériques. Vers une nouvelle sociabilité ? Seuil, Paris, 2010.

[DACHEUX 18] DACHEUX E., L'idéologie numérique contre le sens critique. 2018 〈https://theconversation.com/debatlideologie-numerique-contre-le-sens-critique-94005>

[DEJOURS 03] DeJours C., L'Évaluation du travail à l'épreuve du réel : Critique des fondements de l'évaluation. INRA, Paris, 2003.

[DESACHY 08] DeSAChY B., De la formalisation du traitement des données stratigraphiques en archéologie de terrain. Thèse en Sciences de l'Homme et Société. Université Panthéon-Sorbonne - Paris I, 2 vol. (2008) 〈http://tel.archives-ouvertes.fr/tel-00406241v2〉

[DESACHY 16] DESACHY B., Du carnet de fouilles aux systèmes d'information archéologiques de terrain : quelques remarques sur l'évolution de l'enregistrement et l'impact de l'informatisation. Colloque « Archivage, publication et mise à disposition de données archéologiques » du consortium MASA, 26 et 27 septembre 2016 à la Nanterre, Maison Archéologie et Ethnologie : https://vimeo.com/189334301

[DoueIhi 13] DoueIHI M., Qu'est-ce que le numérique ? PUF, Paris, 2013.

[DUBAR 91] DuBAR C., La socialisation, construction des identités sociales et professionnelles. Armand Colin, Paris, 1991.

[DUBAR 00] Dubar C. La crise des identités. L'interprétation d'une mutation. PUF, Paris, 2000.

[EDgerton 13] Edgerton D., Quoi de neuf? Du rôle des techniques dans l'histoire globale. Le Seuil, Paris, 2013.

[ELLUL 12] ElLUL J., Le bluff technologique. Hachette, Paris, 2012.

[EUSÈBE 19] EuSÈBE S., Imagerie numérique et représentation des données en archéologie », In Situ, n 39, 2019; DOI : https://doi.org/10.4000/insitu.21467

[FERNANDES 13] FERNANDES P., TUFFERY C., BINDER D., BRESSY-LEANDRI C., BRACCO J-P. ET AL., Les formations à silex dans le Sud de la France : Élaboration en multipartenariat d'une base de données géoréférencées, premiers résultats. Actes de la séance de la Société préhistorique française, Nice, 28-29 mars 2013, Société préhistorique française, pp.137-150, Séances de la Société préhistorique française, 2016, 〈hal-01436404〉.

[FILIPPOVA 19] FiLIPPOVA D. Technopouvoir. Dépolitiser pour mieux régner. Les liens qui libèrent, Paris, 2019.

[Foucault 69] Foucault M., L'Archéologie du savoir. Gallimard, Paris, 1969.

[Foucault 04] Foucault M., Sécurité, Territoire, Population, EHESS, Gallimard, Le Seuil, Paris, 2004.

[GARDIN 91] GARDIN J-C., Une contribution des « humanités » à l'informatique : de PENELOPE (1955) à ZETHOS (1974) et au-delà. Le calcul et la raison. Essais sur la formalisation du discours savant. Ed. de l'EHESS, Paris, 1991.

[Goffman 73] Goffman E., La Mise en scène de la vie quotidienne. Les Editions de Minuit, Paris, 1973. 
[Gollac 00] GollaC M., KRAMARZ F., L'informatique comme pratique et comme croyance. Actes de la recherche en sciences sociales, vol. 134, L'informatique au travail. pp. 4-21, septembre 2000.

[GRUEL 90] GRUEL C., BUCHSENSCHUTZ O., Informatique et archéologie, Les dossiers d'Archéologie, 153, pp.80-83, octobre 1990.

[Hey 09] Hey, T., Tansley, S. AND Tolle, K. The Fourth Paradigm: Data-Intensive Scientific Discovery, Microsoft Research, october 2009.

[Huggett 00] Huggett, J. Computers and Archaeological Culture Change Dans On the Theory and Practice of Archaeological Computing, edited by G. Lock and K. Brown, 5-22. Oxford: Oxford University Committee for Archaeology Monograph 51, 2000.

[Huggett 04] Huggett, J. Archaeology and the New Technological Fetishism. Dans Archeologia e Calcolatori 15: 81-92. 2004. http://www.archcalc.cnr.it/indice/PDF15/05_Hugget.pdf.

[Huggett 20] Huggetr, J. Is Big Digital Data Different? Towards a New Archaeological Paradigm. Journal of Field

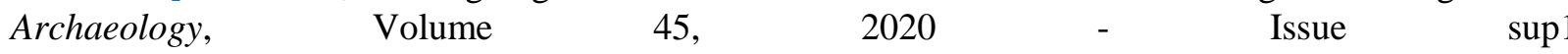
https://www.tandfonline.com/doi/full/10.1080/00934690.2020.1713281.

[ЈасОВ 07] JАСОВ C. (dir.), Lieux de savoir. Tome 1, Albin Michel, Paris, 2007.

[ЈАСОВ 11] JАСОВ C. (dir.), Lieux de savoir. Tome 2, Albin Michel Paris, 2011.

[KoEHLer 12] KoEHLER A., TufFÉRY C., Harmonisation des méthodes et outils pour l'information archéologique à l'Inrap : constats, enjeux et perspectives pour un établissement national. Archeologia e Calcolatori, All'Insegna del giglio, 2012, pp.229-238. 〈hal-01853703〉.

[KUHN 08] KuHN T., La structure des révolutions scientifiques. Flammarion, Paris, 2008.

[LE DeUfF 14] LE DeUfF O. (dir.), Le temps des humanités digitales : la mutation des sciences humaines et sociales. Edition FYP, Paris, 2014.

[LERHOËFF 19] LERHOËFF A., L'archéologie. PUF, Paris, 2019.

[Marcellini 99] Marcellini A., Miliani M., Lecture de Goffman. Corps et culture, Numéro 4, 1999 : http://journals.openedition.org/corpsetculture/641.

[MAUSS 13] MAUSS M., Les techniques du corps. Sociologie et anthropologie, PUF, Paris, 2013.

[Monod 19] MonOD J-C., L'art de ne pas être trop gouverné. Le Seuil, Paris, 2019.

[Morozov 14] Morozov E., Pour tout résoudre cliquez ici : L'aberration du solutionnisme technologique. FYP éditions, Paris, 2014.

[MOUNIER 12] MOUNIER P. (dir.), Read/Write Book 2 : une introduction aux humanités numériques. OpenEdition Press, Marseille, 2012.

[PETIT 19] PETIT A., La recherche, une arme pour les combats du futur, 2019, http://www.lesechos.fr/ideesdebats/sciences-prospective/la-recherche-une-arme-pour-les-combats-du-futur-1150759.

[PETIT 19] PETIT F-X., Il est temps que la technologie numérique prenne conscience de son pouvoir social, 2019, http://www.lemonde.fr/idees/article/2019/12/31/il-est-temps-que-la-technologie-numerique-prenne-conscience-deson-pouvoir-social_6024440_3232.html.

[Py 91] Py M. (dir.), Système d'enregistrement, de gestion et d'exploitation de la documentation issue des fouilles de Lattes. Lattara 4, Lattes, 1991, 224 p.

[Rosa 10] Rosa H., Accélération. Une critique sociale du temps. Ed. La Découverte, Paris, 2010.

[Rosa 20] Rosa H., Rendre le monde indisponible. Ed. La Découverte, Paris, 2020.

[Rouvroy 09] Rouvroy A., Berns T., Le corps statistique. P. Daled, Bruxelles, 2009.

[SADIN 16] SADIN E., La siliconisation du monde. L'irrésistible expansion du libéralisme économique. Ed. L'Echappée, Paris, 2016.

[SERRES 13] SERRES M., Vers de nouvelles sciences humaines? Inria, Objectif 2020. Plan stratégique 2013- 2017, Paris, 2013, p. 4-5

[Simondon 89] Simondon G., Du mode d'existence des objets techniques. Aubier, Paris, 1989. 
[SOULE 07] SOULE B., « Observation participante ou participation observante ? Usages et justifications de la notion de participation observante en sciences sociales ", Revue Recherches Qualitatives, Vol. 27(1), pp. 127-140. http://www.recherche-qualitative.qc.ca/documents/files/revue/edition_reguliere/numero27(1)/soule.pdf

[TUfFÉRY 19a] TufFÉRY C., AugRY S., «Harmonisation de l'acquisition des données d'opérations d'archéologie préventive. Retours d'expériences et perspectives à partir de l'application EDArc ». Atelier DAHLIA DigitAL Humanities and cuLtural herITAge : data and knowledge management analys, Jan 2019, Metz, France. 〈hal02472817).

[TUFFÉRY 19b] TUFFÉRY C., Les compétences numériques en archéologie : un défi majeur et des risques de déni, Revue ¿Interrogations?, $n^{\circ} 28$. Autour du déni, juin 2019, http://www.revue-interrogations.org/Les-competencesnumeriques-en.

[TUFFÉRY 20] TUFFÉRY C., Visibilité et invisibilité des savoirs et savoir-faire en archéologie. Le cas des carnets de terrain et des journaux de fouille, Mémoire de master 2 à l'EHESS, Parcours Histoire des sciences, technologies, sociétés. Mention "Savoirs en sociétés", 155 pages, non publié.

[VERGNieuX 18] VergnieuX R., GiLigny F., « Pour un usage raisonné de la 3D en archéologie », Les nouvelles de l'archéologie, $\mathrm{n}^{\circ} 146,2016$, DOI : https://doi.org/10.4000/nda.3818

[WAquet 15] Waquet F., L'ordre matériel du savoir. Comment les savants travaillent, XVIe-XXIe siècles. CNRS Éditions, Paris, 2015.

[WAQUET 19] WAQUET F., Une histoire émotionnelle du savoir. XVIIe-XXIe siècles. CNRS Éditions, Paris, 2019. 